\title{
Simvastatin attenuates oleic acid-induced oxidative stress through CREB-dependent induction of heme oxygenase- 1 in renal proximal tubule cells
}

\author{
Meaghan Barnett ${ }^{1}$, Samuel Hall ${ }^{2}$, Mehul Dixit ${ }^{2}$ and Istvan Arany ${ }^{2}$
}

BACKGROUND: Statins elicit antioxidant effects independently of their lipid-lowering properties. Heme oxygenase-1 (HO-1) induction may be a part of these pleiotropic effects, which are insufficiently described in the kidney. We hypothesize that simvastatin (SIM) transcriptionally activates $\mathrm{HO}-1$ that protects renal proximal tubule cells from lipotoxic injury.

METHODS: Impact of SIM on $100 \mu \mathrm{mol} / \mathrm{I}$ oleic acid (OA)mediated reactive oxygen species (ROS) production and consequent oxidative stress (4-hydroxynonenal (HNE) content) as well as cell injury/apoptosis (lactate dehydrogenase (LDH) release, caspase-3 activation) were determined in cultured renal proximal tubule (NRK52E) cells. Effect of SIM on the HO-1 promoter and its enhancer elements (antioxidant response element (ARE), CCAAT, AP1, and CAMP response element (CRE)) was also determined in reporter luciferase assays. Dominant-negative (dnMEK, M1CREB) and pharmacologic (H89) approaches were used to inhibit activation of extracellular signal regulated kinase (ERK), CREB, and protein kinase A (PKA), respectively.

RESULTS: SIM dose-dependently activated the $\mathrm{HO}^{-1}$ promoter that was essential for protection against OA-dependent ROS production/oxidative stress and LDH release/caspase-3 activation. We found that the HO-1 promoter was induced through ERK and PKA-dependent activation of the CRE by SIM. CONCLUSION: SIM may protect the kidney from adverse effects of circulating fatty acids by upregulating the antioxidant $\mathrm{HO}-1$, aside from its well-described lipid-lowering effects.

O besity is a growing problem among patients of all ages $(1,2)$ that has a variety of effects on different organs including the kidney (3). Adverse effects of obesity are due to increased vascular reactivity, inflammation, and other factors called lipotoxicity (3). Obesity is associated with an elevation of circulating free fatty acids that are responsible for increased oxidative stress and subsequent lipotoxicity (3). Oleic acid (OA), a free fatty acid, is present in high concentration in the plasma of obese individuals (4). Previously, we showed that OA increases production of reactive oxygen species (ROS) that induces oxidative injury in renal proximal tubule cells (5).
Statins-such as simvastatin (SIM) - are extensively used to lower dyslipidemia in children (6). Statins are not only lower lipids but also shown to exert "pleiotropic" effects that are not due to their lipid-lowering activity (7). For example, SIM transcriptionally activates the antioxidant $\mathrm{HO}-1$ gene in the liver and NIH3T3 cells $(8,9)$. It is plausible that such antioxidant properties can be used to diminish lipotoxic oxidative stress, caused by obesity. HO-1 induction-dependent renoprotective effects of SIM in a rat model of acute ischemia/reperfusion injury are noted (10) but the underlying mechanism and its potential role in obesity is unknown.

Heme oxygenase-1 (HO-1) elicits adaptive responses against oxidative stress in the kidney (11). Oxidative stress transcriptionally induces the $\mathrm{HO}-1$ promoter mostly via the antioxidant response element (ARE) (12). Studies have shown that $\mathrm{HO}-1$ protects from obesity-associated oxidative stress (13) and hence, from lipotoxic injury. Some studies have also shown that SIM increases activity of the ARE and expression of $\mathrm{HO}-1$ protein $(8,14)$; however, there is little data on renal cells.

Accordingly, we hypothesized that SIM protects renal proximal tubule cells from oleic acid-induced oxidative stress via transcriptional upregulation of $\mathrm{HO}-1$.

\section{RESULTS}

SIM Dose-Dependently Induces the HO-1 Promoter but Also Increases Cytotoxicity in Renal Proximal Tubule Cells

First, we needed to determine whether SIM activates the HO-1 promoter and elicits any cytotoxicity. Accordingly, NRK52E cells were transfected with an HO-1-promoter luciferase reporter together with a renilla luciferase and were treated with SIM in doses of $0,5,10,25,50$, and $100 \mu \mathrm{mol} / \mathrm{l}$. Twenty-four hours later, luciferase activities were determined. As shown in Figure 1, panel a, SIM dose-dependently induced the HO-1 promoter but at concentrations higher than $25 \mu \mathrm{mol} / \mathrm{l}$ also significantly increased lactate dehydrogenase $(\mathrm{LDH})$ release (Figure 1, panel b) and ROS production (Figure 1, panel c) indicating toxicity. Hence, $10 \mu \mathrm{mol} / \mathrm{l}$ of SIM dose was chosen for further experiments, which is nontoxic but still significantly 
a

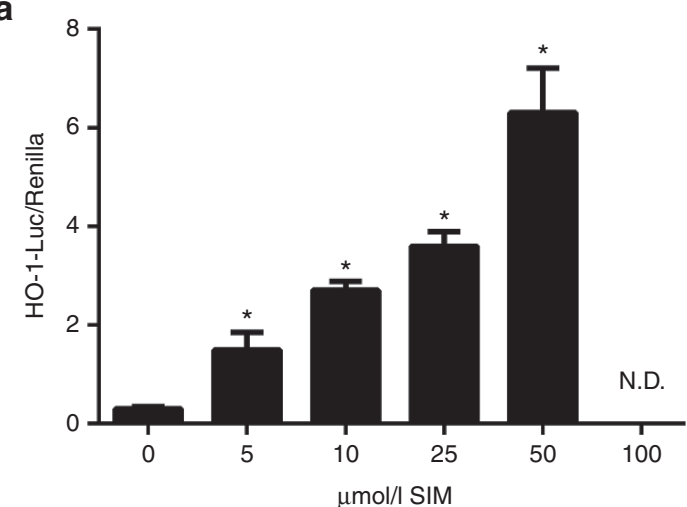

b

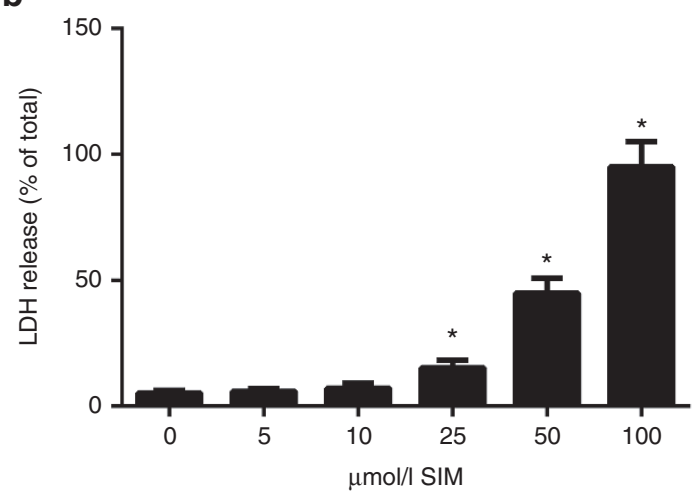

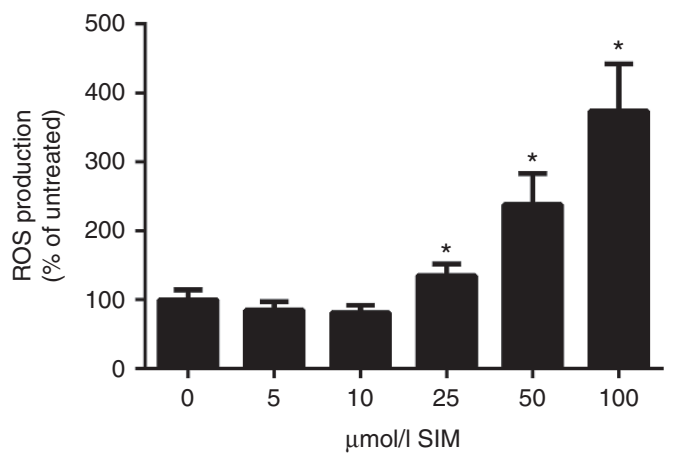

d

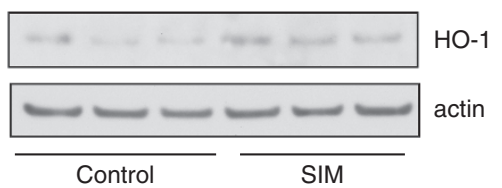

e

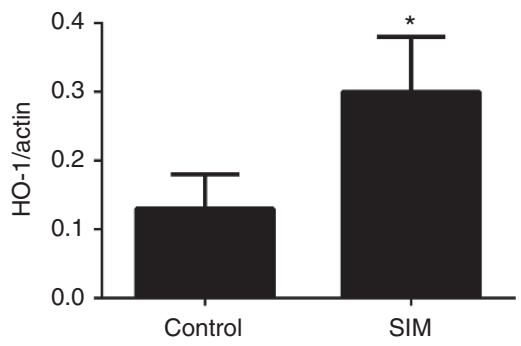

Figure 1. Simvastatin activates HO-1 in renal proximal tubule cells. (a) NRK52E cells were cotransfected with an HO-1 promoter-luciferase and renilla luciferase plasmids and treated with increasing amount of SIM as noted. Luciferase activities were determined as described in Materials and Methods. $n=3 ;{ }^{*} P<0.05$ compared to untreated, N.D., not detectable due to extensive cell death. (b) Cell injury (by means of LDH release) was determined after treatment of NRK52E cells with various concentrations of SIM. $n=3,{ }^{*} P<0.05$ compared to untreated. (c) ROS production was determined after treatment of NRK52E cells with various concentrations of SIM. $n=3,{ }^{*} P<0.05$ compared to untreated. (d) NRK52E cells were treated with $10 \mu \mathrm{mol} / \mathrm{I} \mathrm{SIM} \mathrm{for} 24 \mathrm{~h}$ and protein levels of HO-1 were determined by western blotting. Equal protein loading was assessed by reprobing the blot with an anti-actin antibody. (e) Densitometric analysis of the blots shown in (d). $n=3,{ }^{*} P<0.05$ compared to control (untreated). LDH, lactate dehydrogenase.

increases the activity of the $\mathrm{HO}-1$ promoter and $\mathrm{HO}-1$ protein level (Figure 1, panel a and panels d,e).

\section{SIM Pretreatment Attenuates OA-Dependent ROS Production and Injury in Renal Proximal Tubule Cells That Contingent on HO-1}

Next, we determined whether SIM exerts protective effect against OA-mediated lipotoxicity in renal proximal tubule cells? Accordingly, cells were left untreated or pretreated with $10 \mu \mathrm{mol} / \mathrm{l}$ SIM for overnight prior to treatment with $100 \mu \mathrm{mol} / \mathrm{l} \mathrm{OA}$ : ROS production as well as the resultant oxidative stress was determined. Figure 2, panel a shows, that OA greatly increases ROS production, which was significantly attenuated upon pretreatment with SIM. In addition, 4-hydroxynonenal (HNE) content (a product of lipid peroxidation) of OA-treated cells was also significantly increased (Figure 2, panel b), which indicates increased oxidative stress in OA-treated cells. Importantly, SIM treatment attenuated both ROS production (Figure 2, panel a) and oxidative stress (Figure 2, panel b).
Previously, we demonstrated that OA-associated oxidative stress is responsible for cell injury in cultured renal proximal tubule cells (5). Hence, we determined whether SIM mitigates OA-mediated cell injury? To verify this, cells were pretreated with $10 \mu \mathrm{mol} / \mathrm{l}$ SIM overnight prior to treatment with $100 \mu \mathrm{mol} / \mathrm{l} \mathrm{OA}$ and LDH release was determined $24 \mathrm{~h}$ later. Figure 2, panel $c$ depicts that SIM significantly attenuated LDH release. We also verified that the observed lipotoxicity is-at least partly-due to apoptotic events, extent of which is quantified by measuring caspase $3 / 7$ activity (Figure 2, panel d).

Next, we assessed whether protective effects of SIM are related to induction of HO-1. Accordingly, NRK52E cells were pretreated with $10 \mu \mathrm{mol} / \mathrm{l} \mathrm{SnPP}$ (to inhibit HO-1 activity) $1 \mathrm{~h}$ prior to overnight treatment with $10 \mu \mathrm{mol} / \mathrm{l}$ SIM followed by $100 \mu \mathrm{mol} / \mathrm{l} \mathrm{OA}$. ROS production, intracellular HNE content, LDH release, and caspase 3/7 activation were determined as above. Figure 2, panels a-d show that $\mathrm{SnPP}$ significantly attenuated protective effects of SIM suggesting that HO-1 plays an important role in beneficial effects of SIM. 
a

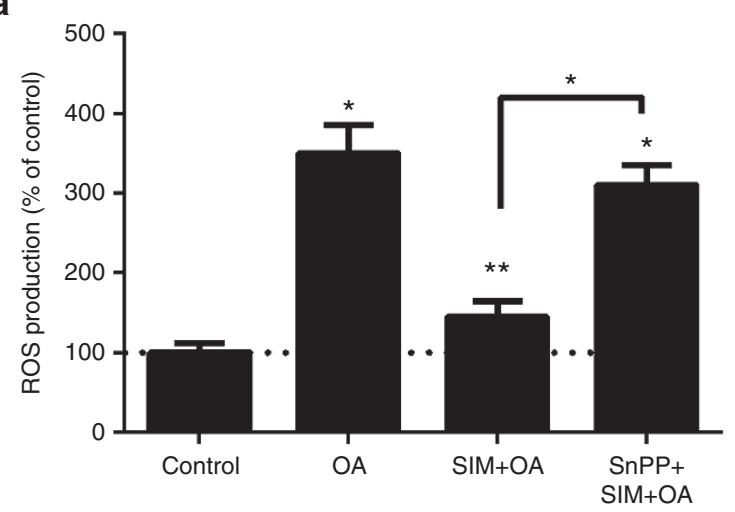

b



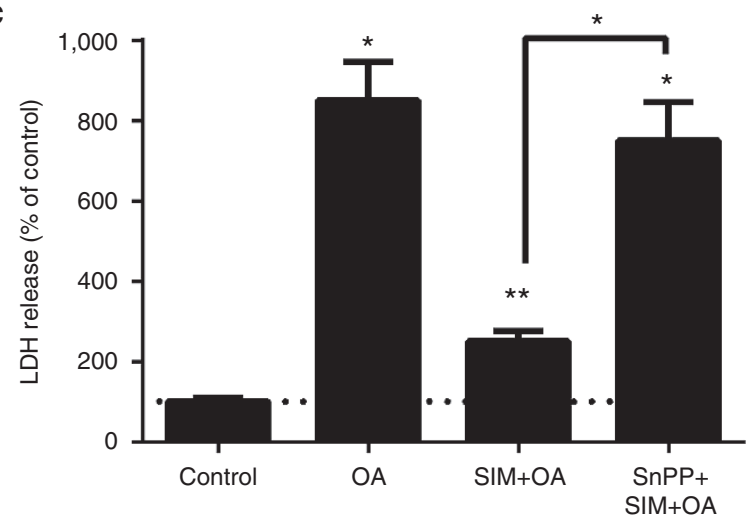

d

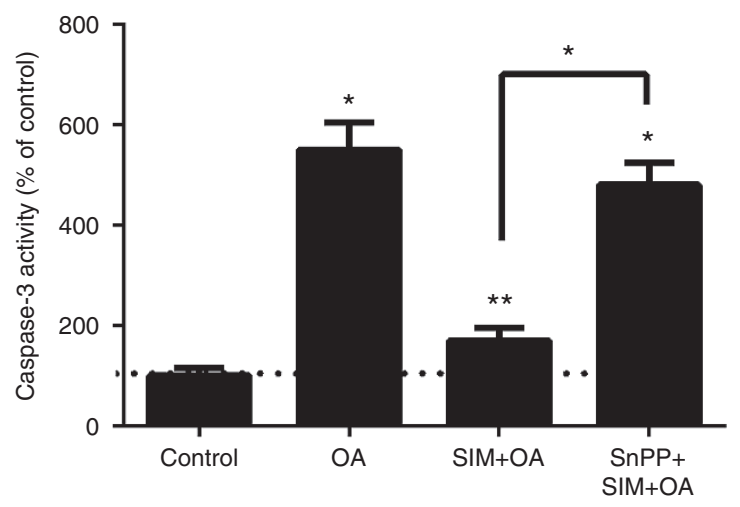

Figure 2. Simvastatin protects renal proximal tubule cells from lipotoxicity via induction of HO-1. (a) NRK52 cells were treated with $100 \mu \mathrm{mol} / \mathrm{I}$ OA and ROS production was determined as described in Materials and Methods. In some experiments, cells were pretreated with $10 \mu \mathrm{mol} / \mathrm{I}$ SIM overnight prior to treatment with OA. To demonstrate the role of HO-1 in SIM-associated protection, $10 \mu \mathrm{mol} / \mathrm{I} \mathrm{SnPP} \mathrm{(HO-1} \mathrm{activity} \mathrm{inhibitor)} \mathrm{was} \mathrm{applied} 30$ min prior to treatment with $10 \mu \mathrm{mol} / \mathrm{I} \mathrm{SIM}$. ROS production was expressed as percentage of untreated. $n=3, * P<0.05$ compared to untreated or as indicated, **P 0.05 compared to OA-treated. (b) Cells were treated similar to (a) and 4-hydroxynonenal (HNE) content of the cells were determined as described in Materials and Methods. HNE content was expressed as percentage of untreated. $n=3,{ }^{*} P<0.05$ compared to untreated or as indicated, ${ }^{* *} P<0.05$ compared to OA-treated. (c) Extent of cell injury was determined by means of lactate dehydrogenase (LDH) release as described in Materials and Methods. $\mathrm{LDH}$ release was expressed as percentage of untreated. $n=3,{ }^{*} P<0.05$ compared to untreated or as indicated, ${ }^{* *} P<0.05$ compared to OA-treated. (d) Presence and extent of apoptosis was determined by means of caspase 3/7 activation as described in Materials and Methods. Caspase 3/7 activation was expressed as percentage of untreated. $n=3,{ }^{*} P<0.05$ compared to untreated or as indicated, ${ }^{* *} P<0.05$ compared to OA-treated.

\section{SIM Activates the HO-1 Promoter Via the CRE but Not the ARE}

Next, we assessed whether ARE, which is a major enhancer in the HO-1 promoter (12), is activated by SIM. Accordingly, NRK52E cells were transfected with an ARE-luciferase reporter together with a renilla luciferase and treated with $10 \mu \mathrm{mol} / \mathrm{l}$ SIM. After $24 \mathrm{~h}$, luciferase activities were determined. Figure 3, panel a shows that-surprisingly-SIM does not activate the ARE even though it induces the HO-1 promoter (Figure 1, panel a). These results suggest that SIM may target other enhancer sites in the HO-1 promoter such as AP-1, CCAAT, or cAMP response element (CRE) (15). Accordingly, NRK52E cells were cotransfected with either of those luciferase reporters plus a renilla luciferase and treated with $10 \mu \mathrm{mol} / \mathrm{l}$ SIM for $24 \mathrm{~h}$. Figure 3, panel a demonstrates that only the CRE was significantly induced by SIM. These results suggest that HO-1 is activated via CRE but not ARE, CCAAT, or AP-1. To prove this, NRK52E cells were cotransfected with a dominant-negative CREB (M1CREB) plasmid (to inhibit CRE activation) together with the HO-1-promoter luciferase plus renilla luciferase and treated with $10 \mu \mathrm{mol} / \mathrm{l}$ SIM. Since M1CREB inhibits SIM-dependent activation of the HO-1 promoter (Figure 3, panel b), consequently, SIM activates $\mathrm{HO}-1$ via CRE.

\section{SIM Activates the CRE and Hence, HO-1, via ERK and PKA}

The CRE binds the activated transcription factor CREB (cAMP response element binding protein) (16). CREB activation occurs via phosphorylation by kinases such as extracellular signal regulated kinase (ERK) or protein kinase A (PKA) (16). First, we determined whether SIM phosphorylates CREB in NRK52E cells, although reporter studies (Figure 3, panel b) indirectly imply it. Accordingly, cells were treated with $10 \mu \mathrm{mol} / \mathrm{l}$ SIM for $30 \mathrm{~min}$; cell lysates were prepared and CREB phosphorylation was determined by western blotting. Figure 4, panels a,b show that CREB phosphorylation is significant at that time point. In the next set of experiments, we determined whether inhibition of ERK phosphorylation (through dominant-negative inhibition of its upstream kinase MEK: dnMEK) or pharmacologic inhibition of PKA (by H89) affects SIM-dependent induction of the CRE reporter. Accordingly, NRK52E cells were transfected with CRE-luc and renilla plasmids together with a dnMEK plasmid or treated with $10 \mu \mathrm{mol} / \mathrm{l} \mathrm{H} 89 \mathrm{~h}$ prior to 

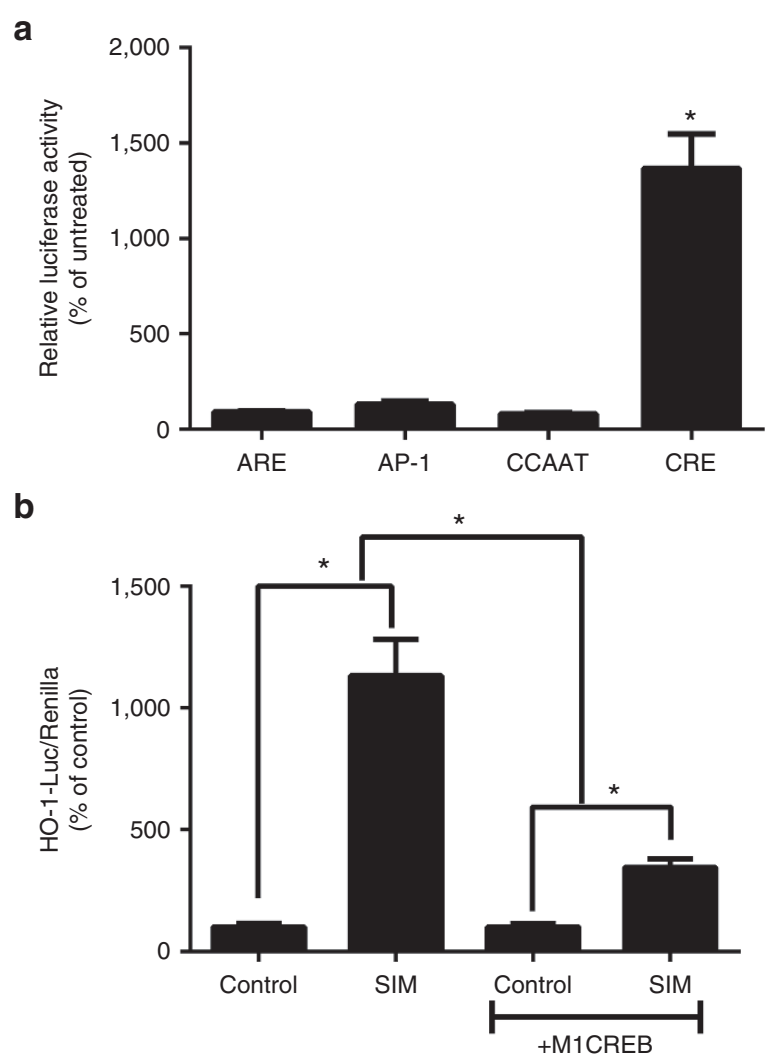

Figure 3. Simvastatin (SIM) activates the heme oxygenase-1 (HO-1) promoter via the CAMP response element (CRE). (a) NRK52E cells were cotransfected with an antioxidant response element, AP-1, CCAAT, or CRE luciferase plasmid together with renilla luciferase and treated with 10 $\mu \mathrm{mol} / \mathrm{I} \mathrm{SIM}$. Twenty-four hours later luciferase activities were determined as described in Materials and Methods. $n=3,{ }^{*} P<0.05$ compared to untreated; M1CREB: mutant CREB (B) NRK52E cells were cotransfected with a HO-1 promoter and renilla luciferase plasmid with or without an M1CREB plasmid. After treatment with $10 \mu \mathrm{mol} / \mathrm{I}$ SIM luciferase activities were determined. $n=3,{ }^{*} P<0.05$ as indicated.

treatment with $10 \mu \mathrm{mol} / \mathrm{l}$ SIM. Luciferase activities were determined $24 \mathrm{~h}$ later. Figure 4, panel c shows that SIM-dependent activation of the CRE reporter was significantly decreased upon inhibition of ERK (dnMEK) or PKA (H89). These results suggest that CRE is activated through ERK and PKA. Not surprisingly, SIM-dependent activation of the HO-1 promoter was also attenuated by dnMEK and H89 (Figure 4, panel d).

\section{Protective Effects of SIM Depends on Activation of ERK, PKA, and CREB}

Our results demonstrate that SIM induces the HO-1 promoter via ERK/PKA-dependent activation of the CRE enhancer. Since protective effects of SIM against lipotoxicity depend on HO-1 activation (Figure 2), it should also depend on activation of ERK, PKA, and CREB/CRE. To prove this, NRK52E cells were transfected with either dnMEK (to inhibit ERK activation), M1CREB (to inhibit CREB) or treated with H89 (to inhibit PKA) prior to treatment with SIM+OA: ROS production, intracellular HNE content, LDH release, and caspase $3 / 7$ activation were determined. Figure 5, panels a-d show that inhibition of ERK, PKA, or CREB significantly abrogated beneficial effects of SIM.
These results suggest that SIM protects from OA-mediated lipotoxicity via ERK/PKA-dependent activation of the CRE enhancer in the HO-1 promoter.

\section{DISCUSSION}

Obesity-linked dyslipidemia is associated with increased oxidative stress in the kidney $(17,18)$. Conversely, treating dyslipidemia with statins preserves renal function $(18,19)$. Interestingly, asides from their lipid lowering effects, statins also elicit antioxidant properties $(10,20)$ that may contribute to the observed renoprotection. It has been shown that statins exert "pleiotropic" properties that are independent from their lipid-lowering effects (7). For example, statins induce HO-1 in nonrenal tissues/cells $(8,9,21-24)$ at the level of transcription in vitro (9) and in vivo (20). However, impact of statins on renal HO-1 is less known: Chen et al. (25) showed that SIM treatment increased renal expression of $\mathrm{HO}-1$, while Gueler et al. (20) showed that cerivastatin increases HO-1 protein and mRNA in the kidney, which was associated with infiltrating macrophages and not renal cells. On the other hand, the mechanism by which SIM regulates renal HO-1 is virtually unknown. Some studies demonstrated that statins activate the ARE (8) or the C/EBP-binding element (CCAAT) $(9,26)$ of the HO-1 promoter in nonrenal cells.

Using NRK52E renal proximal tubule cells, we found that SIM significantly augments activity of a HO-1-promoter luciferase reporter (Figure 1, panel a) and increases expression of HO-1 protein (Figure 1, panels d,e). However, higher concentrations of SIM (above $25 \mu \mathrm{mol} / \mathrm{l}$ ) elicited increasing cytotoxicity (Figure 1, panel b) probably through augmented ROS generation (Figure 1, panel c). Further reporter luciferase studies revealed that SIM activates the CRE but not the ARE, CCAAT, or AP-1 (Figure 3, panel a). Since the dominant-negative M1CREB inhibited SIM-dependent induction of the HO-1 promoter (Figure 3, panel b), we postulated that the CRE is the target for SIM in the promoter proximal region of the HO-1 gene. The CRE element binds the phosphorylated CREB (cAMP response element binding protein) (16) and activates transcription of target genes (27) including the antioxidant HO-1 (12). SIM increases phosphorylation of CREB in the brain (28), in the heart (29), and in endothelial cells (30) while in renal cells or in the kidney is unknown. We discovered that SIM phosphorylates CREB in cultured renal proximal tubule cells (Figure 4, panels a,b). Kinases that phosphorylate CREB include ERK and PKA (16). Inhibitors of ERK (dnMEK) or PKA (H89) inhibited SIM-dependent activation of the CRE (Figure 4, panel c) suggesting that both ERK and PKA are responsible for CREB/CRE activation.

SIM-dependent induction of HO-1 in the kidney (25), lung, and heart (24) of mice has been reported but there is no data available whether this HO-1 induction is CREBdependent. Our in vitro studies reveal that SIM activates the HO-1 promoter via ERK, PKA (Figure 4, panel d), and CRE (Figure 3, panel b). Further in vivo studies are needed to determine impact of SIM on the CREB/HO-1 system in the kidney. Nevertheless, to the best of our knowledge, for the first time 
a

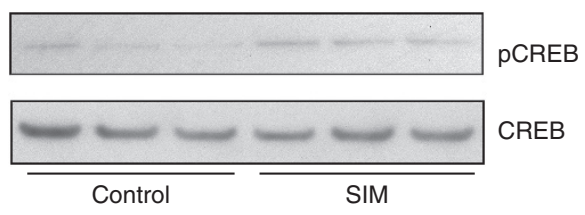

b

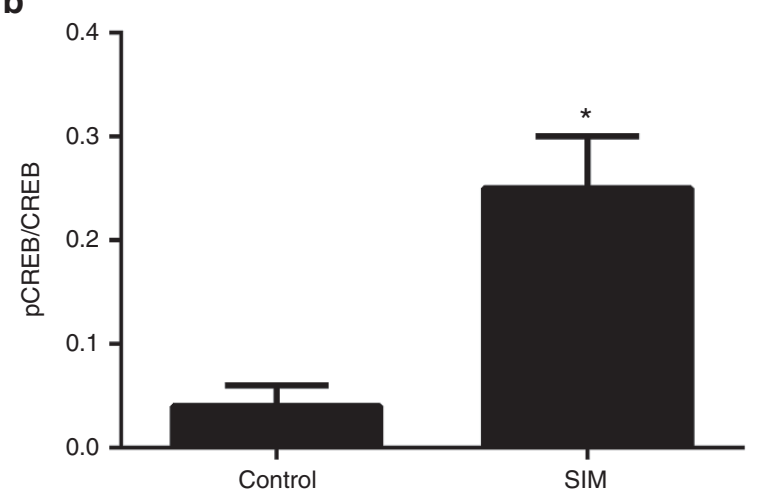

C

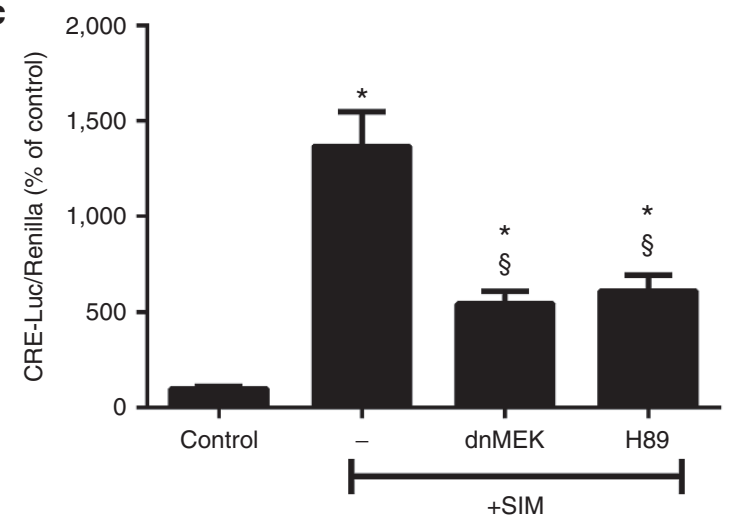

d



Figure 4. Simvastatin (SIM) phosphorylates the CAMP response element (CRE) binding protein CREB in NRK52E cells via extracellular signal regulated kinase (ERK) and PKA. (a) NRK52E cells were treated with $10 \mu \mathrm{mol} / \mathrm{I} \mathrm{SIM} \mathrm{for} 30 \mathrm{~min}$ and CREB phosphorylation was determined by western blotting. Equal loading was determined by reprobing the blot with an anti-CREB antibody. (b) Densitometry of blot from (a). $n=3,{ }^{*} P<0.05$ compared to (untreated) control. (c) NRK52E cells were cotransfected with a CRE and renilla luciferase plasmid in the presence or absence of a dnMEK (to inhibit ERK phosphorylation) or $10 \mu \mathrm{mol} / \mathrm{l} \mathrm{H89}$ (to inhibit PKA activation) and treated with $10 \mu \mathrm{mol} / \mathrm{I} \mathrm{SIM} \mathrm{for} 24 \mathrm{~h}$. Luciferase activities were determined as described above. $n=3$, ${ }^{*} P<0.05$ compared to control, ${ }^{\S} P<0.05$ compared to SIM treated. (d) NRK52E cells were cotransfected with an HO- 1 and renilla luciferase plasmid in the presence or absence of a dnMEK (to inhibit ERK phosphorylation) or $10 \mu \mathrm{mol} / \mathrm{l} \mathrm{H} 89$ (to inhibit PKA activation) and treated with $10 \mu \mathrm{mol} / \mathrm{I}$ SIM for $24 \mathrm{~h}$. Luciferase activities were determined as described above. $n=3,{ }^{*} P<0.05$ compared to control, ${ }^{,} P<0.05$ compared to SIM treated.

in renal cells SIM-dependent and activation of the ERK/PKA/ CREB/CRE/HO-1 axis has been described.

The activated HO-1 protects against oxidative stress in the kidney $(31,32)$ including obesity-associated renal oxidative stress and subsequent renal dysfunction (33). In our in vitro model, SIM protected renal proximal tubule cells from adverse effects of OA: this protection required activation of $\mathrm{HO}-1$ (Figure 2). Since SIM-mediated HO-1 activation is CREB/ERK/PKAdependent (Figures 3 and 4), protective effects of SIM against OA were also CREB/ERK/PKA-dependent (Figure 5).

Based on our data, we propose the following mechanism (Figure 6): SIM activates ERK, which in turn activates CREB but not Nrf2, C/EBP, or jun/fos. The activated CREB binds the CRE in the promoter of the HO-1 gene and activates its transcription. Simultaneously, SIM also activates PKA, whichsimilar to ERK-further activates CREB and hence, the HO-1 promoter. The increased HO-1 then attenuates oleic aciddependent production of ROS and consequent reduced tubular injury (Figure 6).

Since SIM therapy to treat dyslipidemia in children is approved by the FDA (6), the proposed mechanism may offer therapeutic means beyond lipid lowering to slow obesity-associated kidney injury; that may have profound impact on our state as Mississippi has one of the highest rates of childhood obesity (34) and potentially obesity-associated kidney disease per population in the country. However, further in vivo (mice) studies are needed to ascertain whether the above described in vitro mechanism translates.

\section{METHODS}

\section{Cell Lines and Treatment}

The rat renal proximal tubule cell line (NRK52E) was purchased from American Type Culture Collection (Manassas, VA) and maintained in Dulbecco's Modified Eagle's Medium containing 10\% fetal bovine serum in $5 \% \mathrm{CO}_{2}$ atmosphere. Cells were treated with $100 \mu \mathrm{mol} / \mathrm{l}$ oleic acid (Sigma-Aldrich, ST. Louis, MO) as previously reported (5), $5-100 \mu \mathrm{mol} / 1 \mathrm{SIM}$ (Sigma-Aldrich), $10 \mu \mathrm{mol} / \mathrm{l}$ tin protoporphyrine (SnPP, Sigma-Aldrich) to inhibit HO-1 activity or $10 \mu \mathrm{mol} / \mathrm{l} \mathrm{H} 89$ (Sigma-Aldrich) to inhibit PKA.

In our experiments, we used free-instead of albumin-bound-OA, even though fatty acids present in albumin-bound complexes in vivo (35). However, dissociation from albumin precedes fatty acid uptake (35); thus, intracellular effects of fatty acids are attributed to their free form. Also, this way we could exclude the unwanted effects of albumin toxicity (36). Our pilot studies confirmed that bovine serum albumin (BSA) itself increases ROS production and ROS production by BSAconjugated OA is higher than free OA itself (Supplementary Figure S1a 
a

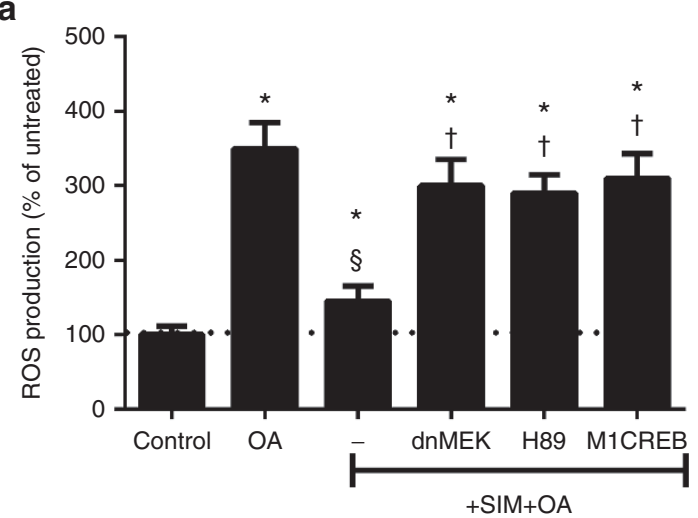

b

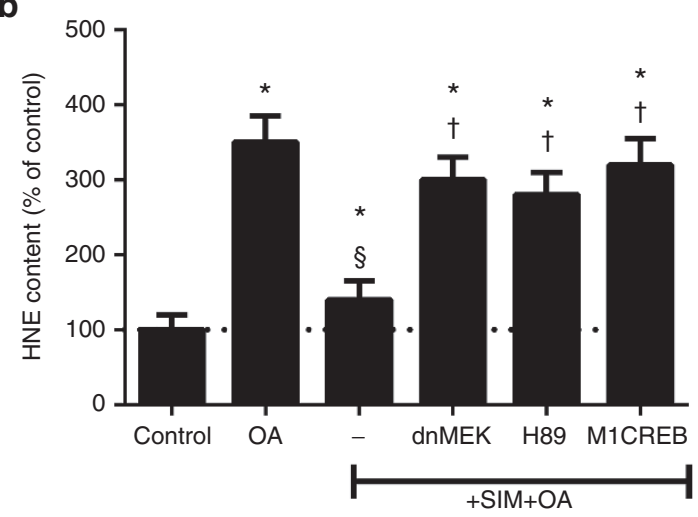

C

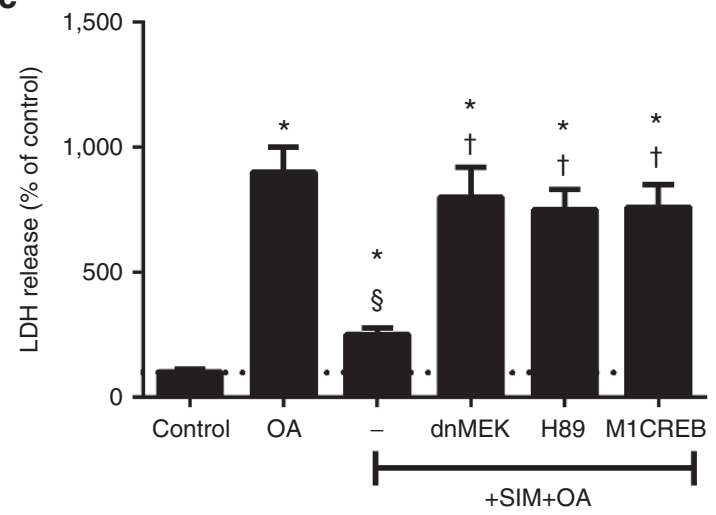

d

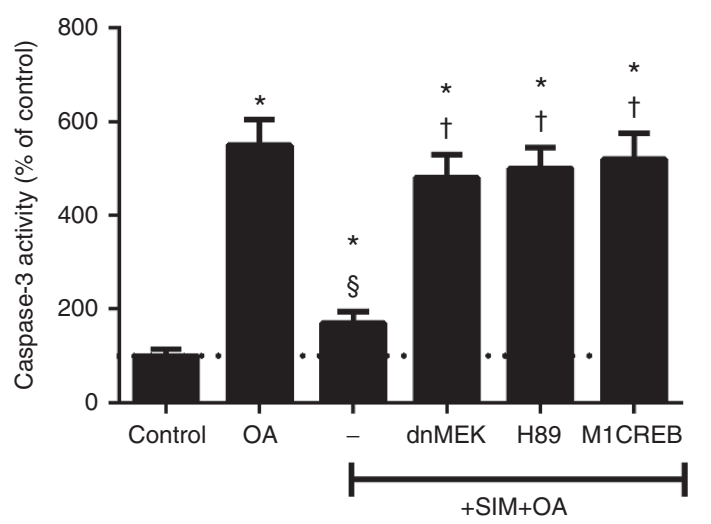

Figure 5. Protective effects of simvastatin (SIM) depends on extracellular signal regulated kinase, PKA, and CREB activation. (a) NRK52E cells were treated with $100 \mu \mathrm{mol} / \mathrm{l}$ oleic acid (OA) in the presence or absence of $10 \mu \mathrm{mol} / \mathrm{I} \mathrm{SIM}$. Some cells were transfected with either dnMEK or M1CREB or treated with $\mathrm{H} 89$ prior to treatment with SIM+OA. Reactive oxygen species production was determined as described in Materials and Methods. Results were expressed as percentage of untreated. $n=3,{ }^{*} P<0.05$ compared to control, ${ }^{\circledR} P<0.05$ compared to OA-treated, ${ }^{\dagger} P<0.05$ compared to SIM+OAtreated. (b) In a similar setting to (a) extent of oxidative stress (intracellular HNE content) was also determined. Results were expressed as percentage of untreated. $n=3,{ }^{*} P<0.05$ compared to control, ${ }^{5} P<0.05$ compared to OA-treated, ${ }^{\dagger} P<0.05$ compared to SIM+OA-treated. In addition, cytotoxicity (by means of lactate dehydrogenase (LDH) release and caspase $3 / 7$ activation) was also determined (c and d). Results were expressed as percentage of untreated. $n=3,{ }^{*} P<0.05$ compared to control, ${ }^{5} P<0.05$ compared to OA-treated, ${ }^{\dagger} P<0.05$ compared to SIM+OA-treated.

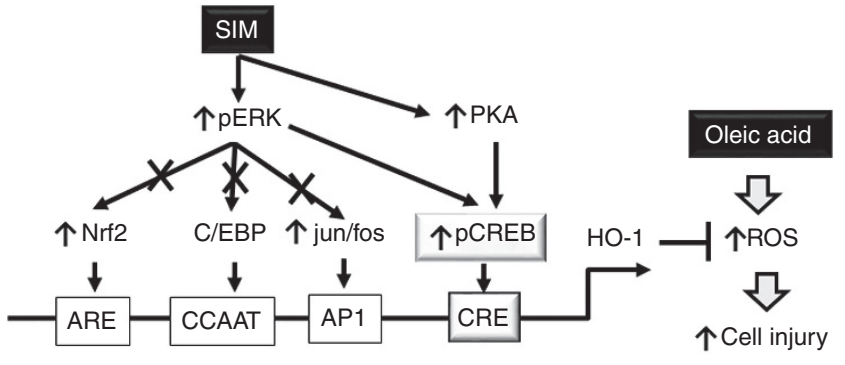

Figure 6. Proposed mechanism for simvastatin (SIM)-mediated activation of the heme oxygenase-1 (HO-1) promoter. SIM activates extracellular signal regulated kinase (ERK), which in turn activates CREB but not Nrf2, $\mathrm{C} / \mathrm{EBP}$, or jun/fos. The activated CREB binds the CAMP response element (CRE) in the promoter of the HO-1 gene and activates its transcription. Simultaneously, SIM also activates PKA, which—similar to ERK-further activates CREB and hence, the HO-1 promoter. The increased HO-1 than attenuates oleic acid-dependent production of reactive oxygen species and consequent tubular injury.

online). In addition, $\mathrm{OA}$ is the predominant free fatty acid in obesity (4): in spite of being a mono-unsaturated fatty acid, it exerts renal lipotoxicity via induction of ROS production $(5,36)$, extent of which is comparable to the saturated palmitic acid (Supplementary Figure S1b online).

Working with cell lines was approved by the Institutional Biosafety Committee of the University of Mississippi Medical Center.

\section{Measurement of ROS Production}

Intracellular generation of ROS was determined by a microplate assay using the oxidant-sensitive 2',7'-dichlorofluorescein-diacetate (DCFDA-Invitrogen, Grand Island, NY). Cells grown in 96-well plates were pretreated with SIM as needed then loaded with 100 $\mu \mathrm{mol} / \mathrm{l}$ DCFDA in Hank's balanced salt solution for $30 \mathrm{~min}$ at $37^{\circ} \mathrm{C}$. After incubation, the dye was washed away with fresh Hank's balanced salt solution and treated with $100 \mu \mathrm{mol} / \mathrm{l} \mathrm{OA}$ : increase in fluorescence was monitored in a fluorescence plate reader (Fluorocount, Packard) at $485 \mathrm{~nm}_{\text {exc }} / 530 \mathrm{~nm}_{\text {em }}$. ROS production was calculated as changes in fluorescence $/ 30 \mathrm{~min} / 0.5 \times 10^{6}$ cells and expressed as percentage of untreated values as described elsewhere (37).

\section{Measurement of Oxidative Stress}

Extent of oxidative stress was determined in lysates of cells by measuring the lipid peroxidation adduct 4-HNE using the OxiSelect HNE-His adduct ELISA kit from Cell Biolabs, San Diego, CA. Briefly, cells grown in 96-well plates were treated with $100 \mu \mathrm{mol} / 1$ oleic acid in the presence or absence of the appropriate pharmacologic/genetic inhibitors for $24 \mathrm{~h}$. Values were normalized to protein content of the lysates and expressed as percentage of the control (untreated) cells.

\section{Reporter Luciferase Studies}

Cells were transfected with either an HO-1-promoter luciferase (12), ARE-luc/Renilla (Qiagen, Valencia, CA), CRE-luc (Agilent Technologies, Santa Clara, CA), AP-1-luc (Agilent Technologies), or CCAAT-luc/Renilla (Qiagen) plasmid. The HO-1-luc, CRE-luc and AP1-luc plasmids were also cotransfected with a renilla luciferase (Promega, Madison, WI) using Lipofectamine 3000 as suggested 
by the manufacturer (Life Technologies, Grand Island, NY). After treatment (as described in the appropriate section) firefly and renilla luciferase activities were determined by a Dual Luciferase assay kit (Promega) and expressed as firefly/renilla ratios.

\section{Cell Injury Assay}

Cell injury was assessed by the fluorescent "CytoTox-One Homogenous Membrane Integrity" assay kit (Promega). Briefly, cells grown in 24-well plates were treated for $24 \mathrm{~h}$; an aliquot of the growth medium was removed and saved. The monolayer was lysed according to the manufacturer's recommendation and $\mathrm{LDH}$ content was determined by a fluorescent substrate both in the medium and cell lysate. LDH release was calculated as percentage of LDH content in the medium compared to the total LDH content (medium+lysate).

\section{Caspase 3/7 Activation}

Activation of caspase 3/7 was determined by the luminescent Caspase-Glo 3/7 assay (Promega) as recommended by the manufacturer. Briefly, cells grown in 96-well plates treated with $100 \mu \mathrm{mol} / \mathrm{l}$ oleic acid in the presence or absence or appropriate pharmacologic/ genetic inhibitors for $24 \mathrm{~h}$ then mixed with equal volume of lysis/ substrate reagent and incubated for $30 \mathrm{~min}$ at room temperature. Luminescence was determined in a Modulus luminometer (Turner Biosystems, Sunnyvale, CA) and results were expressed as percentage of untreated control.

\section{Plasmid Transfection}

NRK52 cells grown in 24-well plates were transfected with a dominantnegative CREB mutant (M1CREB: a gift from Dr. Greenberg (38)) or with a dominant-negative MEK (dnMEK: a gift from Dr. Weber (39)) mutant in order to inhibit CREB or ERK activation, respectively.

\section{Cell Lysates, Western Blotting}

Sodium dodecyl sulfate/polyacrylamide gel electrophoresis and western blotting were performed by using conventional techniques (40). $50 \mu \mathrm{g}$ of cell lysates were separated on a $4-12 \%$ NuPAGE Novex-BisTris gradient mini gel (Invitrogen) and transferred to a polyvinylidene fluoride membrane by using iBlot (Invitrogen). Blots were hybridized with appropriate primary antibodies, visualized by Pierce ECL Western blotting substrate (Thermo Scientific, Rockford, IL), and exposed to an X-ray film (Midwest Scientific, St. Louis, MO). Films were digitized and analyzed by Un-Scan-It Version 6.1 software (Silk Scientific, Orem, UT). The following antibodies were used: anti-HO-1 (Enzo Life Sciences, Farmingdale, NY), anti-phospho-CREB (Cell Signaling Technologies, Danvers, MA), anti-CREB (Cell Signaling Technologies), and anti-actin (EMD/Millipore, Billerica, MA).

\section{Statistical Analysis}

Continuous variables were expressed as mean + SD. Differences between treated and control groups were determined by a one-way ANOVA analysis with the Holm-Sidak post-hoc test. Differences between means were considered significant if $P<0.05$. All analyses were performed using SigmaStat 3.5 software (Systat, San Jose, CA).

\section{SUPPLEMENTARY MATERIAL}

Supplementary material is linked to the online version of the paper at http:// www.nature.com/pr

\section{ACKNOWLEDGMENTS}

The authors thank Alam for the HO-1 promoter luciferase, Greenberg for the M1CREB, and Weber for the dnMEK construct.

\section{STATEMENT OF FINANCIAL SUPPORT}

This work was supported by a grant to M.B. from the Department of Pediatrics, University of Mississippi Medical Center.

Disclosure: none.

\section{REFERENCES}

1. Savino A, Pelliccia P, Chiarelli F, Mohn A. Obesity-related renal injury in childhood. Horm Res Paediatr 2010;73:303-11.

2. Adelman RD. Obesity and renal disease. Curr Opin Nephrol Hypertens 2002;11:331-5.
3. Weinberg JM. Lipotoxicity. Kidney Int 2006;70:1560-6.

4. Gray DS, Takahashi M, Bauer M, Bray GA. Changes in individual plasma free fatty acids in obese females during fasting and refeeding. Int $\mathrm{J}$ Obes 1991;15:163-8.

5. Arany I, Clark JS, Reed DK, Juncos LA, Dixit M. Role of p66shc in renal toxicity of oleic acid. Am J Nephrol 2013;38:226-32.

6. Bamba V. Update on screening, etiology, and treatment of dyslipidemia in children. J Clin Endocrinol Metab 2014;99:3093-102.

7. Gomez SI, Mihos CG, Pineda AM, Santana O. The pleiotropic effects of the hydroxy-methyl-glutaryl-CoA reductase inhibitors in renal disease. Int J Nephrol Renovasc Dis 2014;7:123-30.

8. Habeos IG, Ziros PG, Chartoumpekis D, Psyrogiannis A, Kyriazopoulou V, Papavassiliou AG. Simvastatin activates Keap1/Nrf2 signaling in rat liver. J Mol Med (Berl) 2008;86:1279-85.

9. Mrad MF, Mouawad CA, Al-Hariri M, Eid AA, Alam J, Habib A. Statins modulate transcriptional activity of heme-oxygenase-1 promoter in $\mathrm{NIH}$ 3T3 Cells. J Cell Biochem 2012;113:3466-75.

10. Teshima CA, Watanabe M, Nakamura SH, Vattimo MD. Renoprotective effect of statin: a ischemia-reperfusion animal model. Rev Bras Ter Intensiva 2010;22:245-9.

11. Nath KA. Heme oxygenase-1: a provenance for cytoprotective pathways in the kidney and other tissues. Kidney Int 2006;70:432-43.

12. Alam J, Cook JL. Transcriptional regulation of the heme oxygenase-1 gene via the stress response element pathway. Curr Pharm Des 2003;9: 2499-511.

13. Hosick PA, Stec DE. Heme oxygenase, a novel target for the treatment of hypertension and obesity? Am J Physiol Regul Integr Comp Physiol 2012;302:R207-14.

14. Chartoumpekis D, Ziros PG, Psyrogiannis A, Kyriazopoulou V, Papavassiliou AG, Habeos IG. Simvastatin lowers reactive oxygen species level by Nrf2 activation via PI3K/Akt pathway. Biochem Biophys Res Commun 2010;396:463-6.

15. Alam J, Cook JL. How many transcription factors does it take to turn on the heme oxygenase-1 gene? Am J Respir Cell Mol Biol 2007;36:166-74.

16. Johannessen M, Delghandi MP, Moens U. What turns CREB on? Cell Signal 2004;16:1211-27.

17. Bobulescu IA. Renal lipid metabolism and lipotoxicity. Curr Opin Nephrol Hypertens 2010;19:393-402.

18. Gyebi L, Soltani Z, Reisin E. Lipid nephrotoxicity: new concept for an old disease. Curr Hypertens Rep 2012;14:177-81.

19. Wahba IM, Mak RH. Obesity and obesity-initiated metabolic syndrome: mechanistic links to chronic kidney disease. Clin J Am Soc Nephrol 2007;2:550-62.

20. Gueler F, Park JK, Rong S, et al. Statins attenuate ischemia-reperfusion injury by inducing heme oxygenase-1 in infiltrating macrophages. Am J Pathol 2007;170:1192-9.

21. Grosser N, Hemmerle A, Berndt G, et al. The antioxidant defense protein heme oxygenase 1 is a novel target for statins in endothelial cells. Free Radic Biol Med 2004;37:2064-71.

22. Hinkelmann U, Grosser N, Erdmann K, Schröder H, Immenschuh S. Simvastatin-dependent up-regulation of heme oxygenase-1 via mRNA stabilization in human endothelial cells. Eur J Pharm Sci 2010;41:118-24.

23. Hsieh CH, Rau CS, Hsieh MW, et al. Simvastatin-induced heme oxygenase- 1 increases apoptosis of Neuro $2 \mathrm{~A}$ cells in response to glucose deprivation. Toxicol Sci 2008;101:112-21.

24. Hsu M, Muchova L, Morioka I, Wong RJ, Schröder H, Stevenson DK. Tissue-specific effects of statins on the expression of heme oxygenase- 1 in vivo. Biochem Biophys Res Commun 2006;343:738-44.

25. Chen HH, Chen TW, Lin H. Pravastatin attenuates carboplatin-induced nephrotoxicity in rodents via peroxisome proliferator-activated receptor alpha-regulated heme oxygenase-1. Mol Pharmacol 2010;78:36-45.

26. Mouawad CA, Mrad MF, Al-Hariri M, et al. Role of nitric oxide and CCAAT/enhancer-binding protein transcription factor in statin-dependent induction of heme oxygenase-1 in mouse macrophages. PLoS One 2013; 8:e64092.

27. Mayr B, Montminy M. Transcriptional regulation by the phosphorylationdependent factor CREB. Nat Rev Mol Cell Biol 2001;2:599-609. 


\section{Articles Barrettet al.}

28. Carloni S, Girelli S, Buonocore G, Longini M, Balduini W. Simvastatin acutely reduces ischemic brain damage in the immature rat via Akt and CREB activation. Exp Neurol 2009;220:82-9.

29. Ye Y, Long B, Qian J, Perez-Polo JR, Birnbaum Y. Dipyridamole with lowdose aspirin augments the infarct size-limiting effects of simvastatin. Cardiovasc Drugs Ther 2010;24:391-9.

30. Lee DK, Park EJ, Kim EK, et al. Atorvastatin and simvastatin, but not pravastatin, up-regulate LPS-induced MMP-9 expression in macrophages by regulating phosphorylation of ERK and CREB. Cell Physiol Biochem 2012;30:499-511.

31. Nath KA. Heme oxygenase-1 and acute kidney injury. Curr Opin Nephrol Hypertens 2014;23:17-24.

32. Sikorski EM, Hock T, Hill-Kapturczak N, Agarwal A. The story so far: Molecular regulation of the heme oxygenase-1 gene in renal injury. Am J Physiol Renal Physiol 2004;286:F425-41.

33. Ndisang JF, Tiwari S. Mechanisms by which heme oxygenase rescue renal dysfunction in obesity. Redox Biol 2014;2C:1029-37.

34. Zhang L, Kolbo JR, Kirkup M, et al. Prevalence and trends in overweight and obesity among Mississippi public school students, 2005-2013. J Miss State Med Assoc 2014;55:80-7.
35. Stremmel W, Pohl L, Ring A, Herrmann T. A new concept of cellular uptake and intracellular trafficking of long-chain fatty acids. Lipids 2001;36: 981-9.

36. Ishola DA Jr, Post JA, van Timmeren MM, et al. Albumin-bound fatty acids induce mitochondrial oxidant stress and impair antioxidant responses in proximal tubular cells. Kidney Int 2006;70:724-31.

37. Arany I, Faisal A, Clark JS, Vera T, Baliga R, Nagamine Y. p66SHCmediated mitochondrial dysfunction in renal proximal tubule cells during oxidative injury. Am J Physiol Renal Physiol 2010;298:F1214-21.

38. Ginty DD, Bonni A, Greenberg ME. Nerve growth factor activates a Rasdependent protein kinase that stimulates $\mathrm{c}$-fos transcription via phosphorylation of CREB. Cell 1994;77:713-25.

39. Weber JD, Raben DM, Phillips PJ, Baldassare JJ. Sustained activation of extracellular-signal-regulated kinase 1 (ERK1) is required for the continued expression of cyclin D1 in G1 phase. Biochem J 1997;326 (Pt 1): $61-8$.

40. Arany I, Faisal A, Nagamine Y, Safirstein RL. p66shc inhibits pro-survival epidermal growth factor receptor/ERK signaling during severe oxidative stress in mouse renal proximal tubule cells. J Biol Chem 2008;283: 6110-7. 